\title{
\begin{tabular}{l|l} 
Mitraries & DSpace@MIT
\end{tabular}
}

\author{
MIT Open Access Articles
}

\section{Submodule Integrated Distributed Maximum Power Point Tracking for Solar Photovoltaic Applications}

The MIT Faculty has made this article openly available. Please share how this access benefits you. Your story matters.

Citation: Pilawa-Podgurski, Robert C. N., and David J. Perreault. “Submodule Integrated Distributed Maximum Power Point Tracking for Solar Photovoltaic Applications." IEEE Trans. Power Electron. 28, no. 6 (n.d.): 2957-2967.

As Published: http://dx.doi.org/10.1109/TPEL.2012.2220861

Publisher: Institute of Electrical and Electronics Engineers (IEEE)

Persistent URL: http://hdl.handle.net/1721.1/86981

Version: Original manuscript: author's manuscript prior to formal peer review

Terms of use: Creative Commons Attribution-Noncommercial-Share Alike 


\title{
Sub-Module Integrated Distributed Maximum Power Point Tracking for Solar Photovoltaic Applications
}

\author{
Robert C.N. Pilawa-Podgurski, David J. Perreault \\ RESEARCH LABORATORY OF ELECTRONICS \\ MASSACHUSETTS INSTITUTE OF TECHNOLOGY \\ Corresponding Author: Robert Pilawa-Podgurski \\ University of Illinois at Urbana-Champaign \\ 351 Everitt Laboratory, 1406 W. Green St, Urbana IL 61801, USA \\ Tel. +1-217-244-0181 \\ E-mail pilawadilinois.edu
}

A shortened version of this manuscript has been submitted to IEEE Energy Conversion Congress and Exposition 2012.

\begin{abstract}
This paper explores the benefits of distributed power electronics in solar photovoltaic applications through the use of sub-module integrated maximum power point trackers (MPPT). We propose a system architecture that provides a substantial increase in captured energy during partial shading conditions, while at the same time enabling significant overall cost reductions. This is achieved through direct integration of miniature MPPT power converters into existing junction boxes. We describe the design and implementation of a high-efficiency $(>98 \%)$ synchronous buck MPPT converter, along with digital control techniques that ensure both local and global maximum power extraction. Through detailed experimental measurements under real-world conditions, we verify the increase in energy capture and quantify the benefits of the architecture.
\end{abstract}

\section{INTRODUCTION}

With rising world-wide energy demands and soaring prices of fossil fuels, interest in renewable energy sources has increased. Among these, solar photovoltaic (PV) energy has seen a rapid growth in the last few years, resulting in decreased prices of PV panels as production capacity increases at a fast pace. As the PV panel prices decrease, the cost of the power electronics required to extract the maximum power of the PV modules and to interface the PV system to the grid is becoming a larger part of the overall system cost [1]. Much attention has therefore been given to the development of power electronics that enable a cost reduction of the overall system. In addition, much research is focused on increasing the efficiency of the power processing stage, as well as on improving the power yield of the overall system [2], [3].

Many PV installations suffer from current mismatch between different panels, due to non-uniform shading of the array, dirt accumulation, or manufacturing variability. Ensuring uniform illumination is particularly challenging in residential PV applications, where large current mismatch can be present due to external objects that cause shading. Shown in Fig. 1a is the most common solar PV architecture, which connects all panels in series. In this architecture, any partial shading or other source of cell current mismatch will cause the overall system output power to be reduced, since the current in the string is limited by the weakest panel. While all panels used today employ bypass diodes that help protect the panels and limit the negative effect of partial shading, any partial shading can still have a significant negative effect on a solar installation [?].

The micro-inverter (also known as panel-level inverter) concept shown in Fig. 1b has been proposed [?] to address this problem by operating each panel at its maximum power point, and perform DC to AC conversion for each panel. While this technique can increase overall energy capture in a system, micro-inverters typically suffer from lower overall efficiency than high-voltage string-level inverters, owing to the large voltage transformation required to interface the panel voltage (e.g. 20-40 V) to the grid (e.g. 120-240 V rms), as well as the need for overall low-cost. In single-phase micro-inverters, the twice line-frequency power ripple also introduces an additional challenge, as this must be buffered at each individual micro inverter, leading to the use of large panel-voltage capacitors [?] or more complicated converter topologies that employ highvoltage capacitors [?].

Recently, the concept of dc-dc optimizers has become popular [4], [5], where each PV panel employs a dc-dc converter that performs maximum power point tracking (MPPT), and the output of the converters are connected in series. This architecture is shown in Fig. 1c.Through dc-dc optimizers, localized control of panel voltage and current can be achieved, and each panel can operate at its independent maximum power point (MPP), thus improving the energy extraction of the overall system. The series connection of the outputs provides an inherent voltage stacking that enables each dc-dc converter to operate at a relatively low voltage conversion ratio (enabling high conversion efficiency), while still achieving high overall output voltage, which is desirable as it enables the use of a central, high-voltage, high-efficiency inverter.

To date, however, the promise of dc-dc optimizers has not been fully realized, primarily due to the difficulty of 


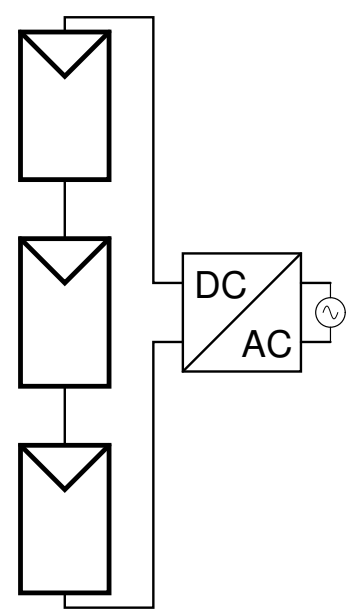

(a) Series string architecture.

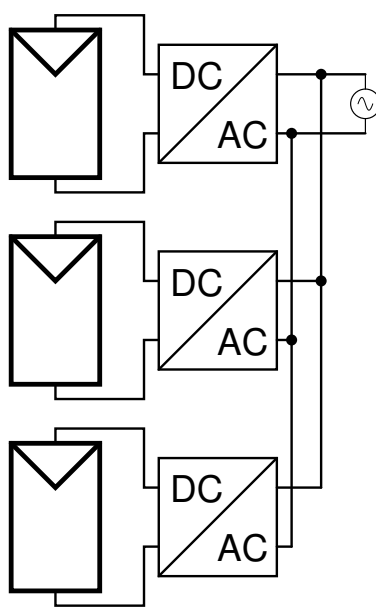

(b) Micro-inverter architecture.

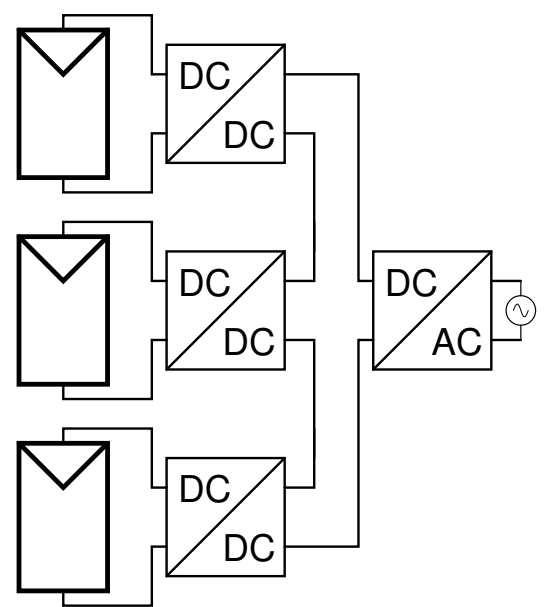

(c) Cascaded dc-dc (dc-dc optimizer) architecture.

Fig. 1. Schematic drawings of three kinds of distributed MPPT architectures for solar PV.

simultaneously achieving high conversion efficiency and low cost of the power electronics. Low dc-dc conversion efficiency can easily negate any increase in energy capture that is offered by more localized (panel-level) control, and must therefore be addressed. Furthermore, a solution that increases total energy capture by a few percent, but which also increases the overall cost by more than the monetary value of the increased power (as compared to the installed system cost) will likely fail in the marketplace. In this paper, we present a dc-dc optimizer system that achieves both low cost and high conversion efficiency, while at the same time capturing substantially more energy than dc-dc optimizer architectures presented to date. In addition, detailed field experiments are presented that illustrate the benefits of our architecture under real-world partial shading conditions.

This paper is organized as follows: Our proposed system is presented in Section II, and Section III provides implementation details of the power converter designed for our architecture. The control implementation is discussed in Section IV, and experimental results and analysis are provided in Section V. A quantitative comparison to previous work is presented in Section VI, where we also introduce a Figure-ofMerit that incorporates cost, efficiency, and increase in energy capture. Finally, Section VIII concludes the paper.

\section{Proposed Architecture}

Dc-dc optimizer systems can be implemented with several different circuit topologies. Previous work at the panel-level has employed boost converters [4] and non-inverting buckboost converters [5]. While boost converters are an attractive option because of their ability to increase the output voltage (requiring fewer panels for a given desired output voltage), their chief disadvantage is their limited operating range. As discussed in [4], since the output current of a boost converter can never be higher than its input current, the range under which current mismatch can be addressed is severely limited.
The non-inverting buck-boost converter is employed in [5], and can provide an output current that is both higher and lower than the input current, thus providing both a voltage increase and the ability to handle shaded panels (although within a limited range, since each converter only operates in buck or boost mode at a given time). The chief disadvantages of the non-inverting buck-boost topology are the increased number of transistors, and the achievable conversion efficiency, which is typically lower than buck or boost converters for the same switch rating.

In this work, we chose to implement the dc-dc optimizer system using synchronous buck converters. While the synchronous buck topology enables both high switching frequency (important for small size, low cost) and high efficiency, it does not contribute any voltage gain (which would reduce the number of panels that must be series connected). In most residential and utility-based installations, however, there are a sufficient number of PV panels to provide for the inherent stacking of voltages without requiring the additional step-up from the power converter. By not tasking the power stage with providing additional voltage step-up, it can be optimized for size, cost, and efficiency. As our experimental results indicate, the synchronous buck converter topology offers size, cost, and efficiency benefits, and the system can be operated in a manner where the control implementation is relatively simple. Meanwhile, the string current can be kept sufficiently low so that the added wiring conduction losses are kept to a minimum.

In order to increase the overall system energy capture, our design employs sub-module distributed MPPTs, as shown in Fig. $2^{1}$. Using this architecture, mismatch between different sub-modules within the same panel can be mitigated, which yields an increase in energy capture compared to panel-level

\footnotetext{
${ }^{1}$ We will refer to all cells in a PV panel that are connected to the same bypass diode as a sub-module. The most common type of PV panels comprise three sub-modules.
} 
TABLE I

COMPONENT LISTING

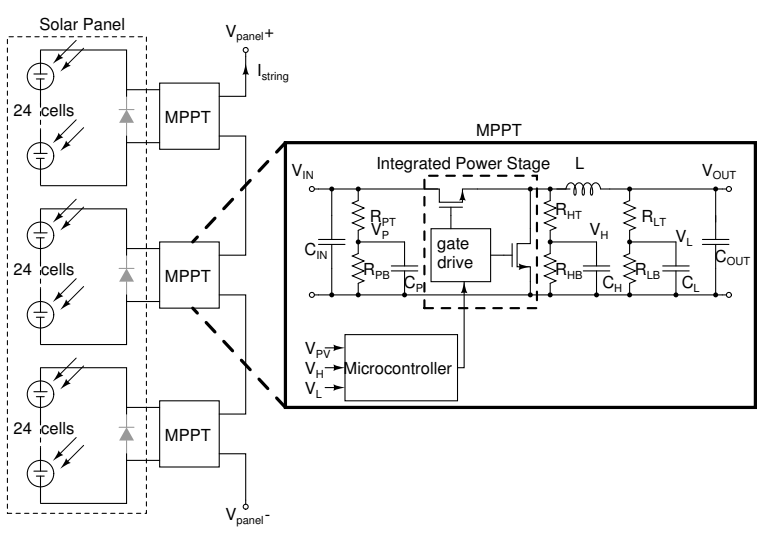

Fig. 2. Schematic drawing of sub-module integrated MPPT system. A component listing is provided in Table I.

MPPTs. Furthermore, each MPPT in Fig. 2 only sees a third of the panel voltage, and can thus be designed using components with lower voltage rating than panel-level MPPTs. The use of low-voltage power MOSFETs with their small parasitics in turn enables an increase in achievable switching frequency, which enables reduced passive component size and cost. As will be shown it is even possible to miniaturize the MPPTs to the point where they can fit in the existing standard junction box at the back of the panel. This leads to further cost reductions, as a large custom outdoor-rated enclosure would contribute significant cost to a dc-dc optimizer system.

\section{Sub-Module Distributed MPPT Converter}

The inset of Fig. 2 shows a schematic drawing of the submodule MPPT architecture designed as part of this work. The system comprises a synchronous buck converter power stage controlled by a microcontroller to achieve local MPP operation. The microcontroller can sense voltage, and also employs lossless current sensing [7] for control algorithms that also require current information. Each converter employs an isolated I2C communication interface, which enables bidirectional information transfer to a master node, which can be a dedicated microcontroller or a computer. It should be noted that each MPPT can operate without any communication interface, but the $\mathrm{I} 2 \mathrm{C}$ interface is used here to gather diagnostic data from each converter, and to provide a simple means for controlling the global output power. Table I provides a listing of the components used in the design. A complete bill-ofmaterial and cost analysis can be found in [6].

Shown in Fig. 3a is a photograph of the complete converter prototype, together with a pencil for scale. Shown in Fig. 3b is one of the MPPTs placed in a typical solar panel junction box. In a full installation, three converters are installed in a single module, one in parallell to each bypass diode. It is evident that the converter fits in the junction box, with plenty of space for connectors and sufficient air-flow. A goal of the power stage design was to achieve a small enough converter footprint

\begin{tabular}{llll}
\hline Device & Model & Value & Manufacturer \\
\hline Integrated Power Stage & FDMF6704A & & Fairchild \\
$\mathrm{L}$ & SER1360-103KL & $10 \mu \mathrm{H}$ & Coilcraft \\
$\mathrm{R}_{H T}, \mathrm{R}_{L T}, \mathrm{R}_{P T}$ & 0402 & $100 \mathrm{k} \Omega$ & Panasonic \\
$\mathrm{R}_{H B}, \mathrm{R}_{L B}, \mathrm{R}_{P B}$ & 0402 & $10 \mathrm{k} \Omega$ & Panasonic \\
$\mathrm{C}_{H}, \mathrm{C}_{L}, \mathrm{C}_{P}$ & 0402 & $1 \mu \mathrm{F}$ & Murata \\
$\mathrm{C}_{I N}$ & $1206, \mathrm{X} 5 \mathrm{R}, 25 \mathrm{~V}$ & $3 \times 10 \mu \mathrm{F}$ & Murata \\
$\mathrm{C}_{O U T}$ & $1206, \mathrm{X} 5 \mathrm{R}, 25 \mathrm{~V}$ & $2 \times 10 \mu \mathrm{F}$ & Murata \\
Microcontroller & ATtiny861 & & Atmel \\
\hline
\end{tabular}

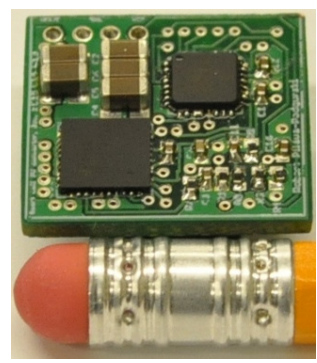

(a) Photograph of the submodule MPPT converter with pencil shown for scale. The power inductor is on the bottom side of the PCB.

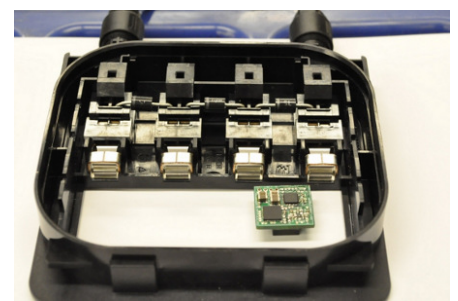

(b) Photograph showing discrete implementation of the power converter together with a solar panel junction box.
Fig. 3. Photographs of sub-module MPPT hardware.

to fit into the junction box on the back of off-the-shelf PV panels. By utilizing the existing weather-resistant junction box as an enclosure, significant cost savings can be realized. The Integrated Power Stage is a combined gate-drive and power MOSFET chip (FDMF6704A), which also incorporates a 5 $\mathrm{V}$ linear regulator, enabling the converter to be completely powered from the sub-module.

In order for the sub-module distributed MPPT architecture of Fig. 2 to be effective, it is important that the additional power captured by more localized control is not wasted by low conversion efficiency of the power electronics. Much care was thus taken in this work to achieve high efficiency operation, both through the choice of topology and passive components, as well as the implementation of sensing and control. A detailed description of these efforts can be found in [6]. Shown in Table II is an overview of the specifications of the converter, along with a performance summary.

A detailed efficiency and power characterization of the MPPT converter has been carried out to measure performance

TABLE II

CONVERTER SPECIFICATIONS

\begin{tabular}{ll}
\hline Input Voltage Range & $5-27 \mathrm{~V}$ \\
Output Voltage Range & $0.8-20$ \\
Max Output Power & $80 \mathrm{~W}$ \\
Switching Frequency & $250 \mathrm{kHz}$ \\
Converter Peak Efficiency & $98.2 \%$ \\
\hline
\end{tabular}




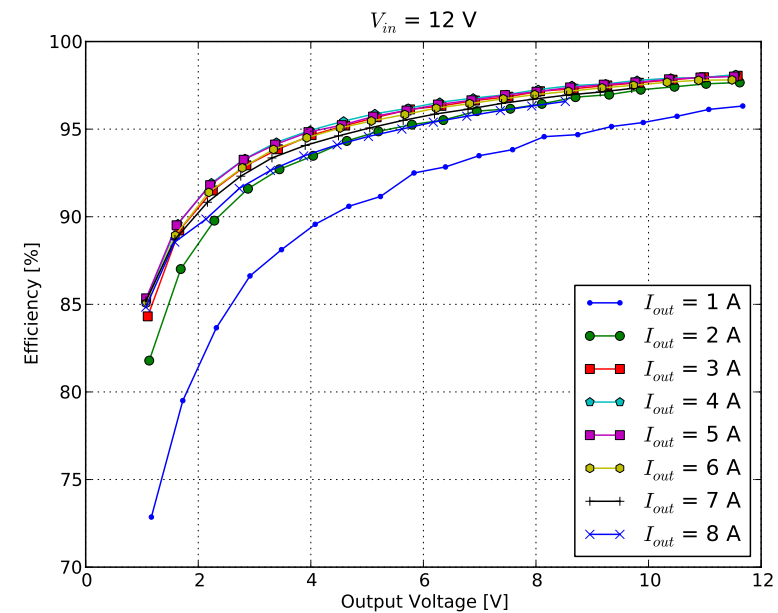

Fig. 4. Measured efficiency versus output voltage, parameterized by output current. A lower output voltage corresponds to a shaded sub-module, while a lower output current signifies a string with less insolation.

across a wide load and output voltage range. Fig. 4 shows a plot of efficiency versus output voltage, parameterized by output current, for a fixed input voltage of $12 \mathrm{~V}$. The converter will operate at lower output voltages if it suffers from more shading relative to the other converters in the string. A low output current would signify that the insolation of the entire string of MPPTs is relatively low. Given these characteristics of the system, it is important to achieve high efficiency at high power levels (for maximum total energy capture), as well as at operating points where the converter is expected to spend significant time in real-world scenarios. In Fig. 4, this would correspond to high output voltage (no or little shading) and high current ( $>5 \mathrm{~A}$, corresponding to high insolation). We see from the plot that we achieve an efficiency above $97 \%$ under these conditions. It should be noted that all efficiency measurements include all sensing, gate drive and control losses, as the converter itself is powered from its input terminals. A more detailed performance characterization of the power stage across a variety of operating conditions can be found in [6].

\section{Control Implementation}

In our architecture, owing to the sub-panel integrated dc$\mathrm{dc}$ converters, there are no local maximas in the I-V characteristics of the overall system (caused by conducting bypass diodes in regular PV panels during partial shading). In order to extract maximum energy from a PV installation with submodule power tracking, each MPPT must continuously operate its sub-module at the correct current and voltage, while also allowing all other MPPTs do the same for their individual sub-modules. We must thus design a control algorithm that ensures that each sub-module operates at its local MPP, while also ensuring that the overall system operates at the global MPP (i.e., the overall string voltage and current are such that all sub-modules are operating at their respective MPPs).

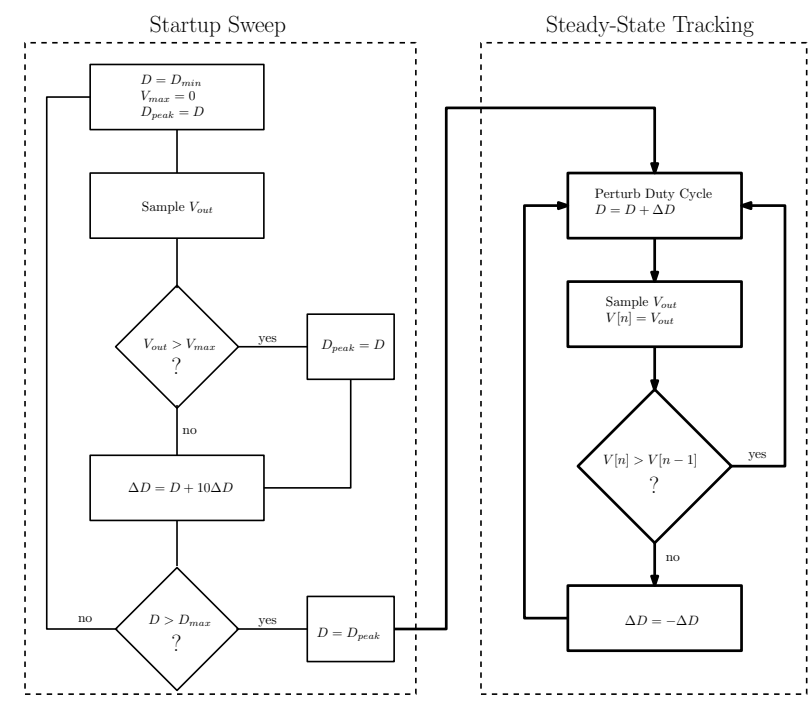

Fig. 5. Flow chart diagram illustrating the local MPPT algorithm. The approximate MPP is first found via a coarse startup sweep, followed by a perturb and observe algrithm that strives to maximize converter output voltage.

\section{A. Local MPPT algorithm}

Since the outputs of the individual power trackers are connected in series (as seen in Fig. 2), all of them share the same output current $\left(I_{\text {string }}\right)$. If the number of seriesconnected converters is large (which is typically the case in a system installation, where a large output voltage is desired), the string current (from the perspective of a single MPPT) can be considered constant. With a constant output current, each converter can then maximize its own output power by maximizing its output voltage. It thus follows that a local MPPT algorithm can be implemented by driving the local output voltage to its maximum value. In our implementation, we employ a Perturb and Observe algorithm that continously tracks the MPP by making small changes to the duty cycle in order to drive the converter output voltage to its maximum. Shown in Figure 5 is a flow chart diagram of the local MPPT algorithm.

In order to quickly locate the approximate location of the MPP, the converter starts by performing a coarse sweep of its duty cycle, and measuring the corresponding values of output voltage. The duty cycle corresponding to the maximum voltage observed is recorded, and at the end of the startup sweep the converter is set to operate at this duty cycle. At this point, the steady-state tracking algorithm begins, which uses a perturb and observe algorithm which aims to maximize the converter output voltage by making small changes $(\Delta D)$ to the duty cycle (D). In this manner, the sub-module MPPT will continuously track the MPP, and oscillate around it to within the finite precision of its voltage sensing and duty cycle control. Table III provides information about our sensing and PWM resolution and step-size in the experimental prototype of this work. 
TABLE III

MPPT TRACKING PARAMETERS

\begin{tabular}{ll}
\hline MPPT Duty Cycle Step-Size & $0.6 \%$ \\
MPPT Startup Sweep Step-Size & $5 \%$ \\
Minimum Duty Cycle & $10 \%$ \\
Maximum Duty Cycle & $99 \%$ \\
ADC Resolution & $10 \mathrm{bit}$ \\
ADC Samples Per Measurement (Overampling) & 100 \\
\hline
\end{tabular}

\section{B. Global MPPT algorithm}

By adjusting the duty ratio, the local MPPT can autonomously achieve MPP operation so long as sub-module current at its MPP is equal to or less than that of the string ${ }^{2}$.

To achieve global MPP operation, each sub-module controller adjusts its duty ratio for MPP operation (e.g., in a "fast" loop) based on the string current, while the system level controller (typically implemented by the grid-interface inverter) adjusts the string current (in a "slow" loop) such that there is just sufficient string current available for the submodule with the highest MPP current. In this manner, the control problem can be separated into a local MPPT control for each sub-module, along with a single global control loop that only requires limited information.

1) 1-bit Feedback Global Algorithm: One method to ensure that the overall system is operating at the global MPP is to signal to the global ("slow") loop controller when one of the local MPPTs operate at its maximum permitted duty cycle. At this point, the system loop controller may not decrease the current $\left(I_{\text {string }}\right)$ any further, as the strongest MPPT would then not be operating at its MPP. This 1-bit feedback signal can be implemented either using a very simple single-interconnect or zero-interconnect communications link, or by encoding the information to communicate it directly via the series string interconnect. (We note that such methods are well known in other types of distributed power conversion systems [10] and can be implemented without significant expense in this application.) One disadvantage of this method is that it would require the global controller (the inverter in a typical installation) to implement this functionality, such that separate dedicated hardware and firmware is required at the inverter level.

2) Communication-less Global Algorithm: It is also conceivable that with the appropriate sub-module level control, global maximum power point operation can be ensured without any communication between individual converters, or between converters and the string-level inverter. All PV inverters used with conventional solar panels today already implement a maximum power point tracking functionality. It would be highly desirable to leverage this existing infrastructure to achieve both global and local optimization with existing inverter hardware.

\footnotetext{
${ }^{2}$ This constraint is due to the chosen power converter topology (buck converter), where the power stage can only increase the output current.
}

If the global MPPT controller draws too little current, the strongest MPP will operate at its maximum duty cycle, and its sub-module will deliver $I_{\text {string, }}$, which will be less than its $I_{m p p}$. Since this sub-module is no longer operating at its individual MPP, the overall output power of the string will decrease. When the global controller detects this decrease in power, it will act to reverse this change, thus increasing the string current. The global MPPT algorithm itself can thus ensure that the string current is not operating at a current that is lower than the highest $I_{m p p}$ of the sub-modules.

The buck-topology can theoretically produce any output current that is higher than its input current (although there are certainly practical limits such as device parasitics, duty cycle resolution, and loss mechanisms that limit the maximum output current). In a real converter, the conduction losses in the MOSFETs, inductor, and wiring will increase as the output current is increased, leading to lower conversion efficiency at very high currents. A lower conversion efficiency in the sub-module MPPTs will lead to lower string power, which can be detected by the global MPPTs algorithm if the output current is increased too much. It should be noted that this effect (decrease in output power by reduced conversion efficiency) is much less pronounced than the relatively sharp drop-off in power observed in a regular PV panel when it operates away from the MPP. The distributed MPPT thus have the effect of significantly "flattening" the power versus voltage (or current) characteristics of the system. The advantage of this is that the central inverter can operate at many different voltage and current levels (while drawing near maximum power from the system). There is, however, a risk that the central inverter may not be able to detect the small changes in power associated with the change in sub-module MPPT efficiency, and may continuously wander across a wide current and voltage range as it searches for the global MPPT.

In the experimental measurements of Section $\mathrm{V}$, we will see the results of a control mechanism that makes use of the 1-bit feedback global MPPT algorithm. The flattening effect of the distributed MPPTs will also be observed, and we can quantify the resolution required to implement the communication-less global MPPT algorithm in practice.

\section{Field Measurement Experimental Results}

In order to fully evaluate the distributed MPPT system in a real setting, we chose to perform outdoor field experiments under a variety of conditions. A PV panel (the STP175S-24/Ab01 72-cell monocrystalline Si panel from Suntech) was mounted in a south-facing direction together with test equipment on a flat roof of a campus building. The camera was used to produce time-lapse photos of the shading pattern of the panel. The photos were synchronized with the output power measurement, which provides a visual check to discern shading patterns related to panel I-V characteristics. Figure 6 shows an annotated photograph of the field setup. The distributed MPPTs were connected across each sub-module (in parallel with the existing junction diodes, as shown in Fig. 2), and their output connected to an electronic load (HP6060B). The 


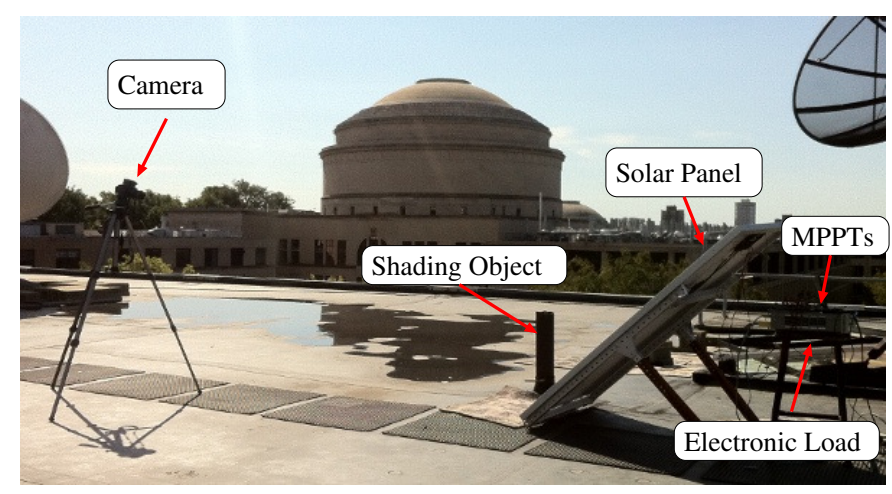

Fig. 6. Annotated photograph of the field experiment setup.

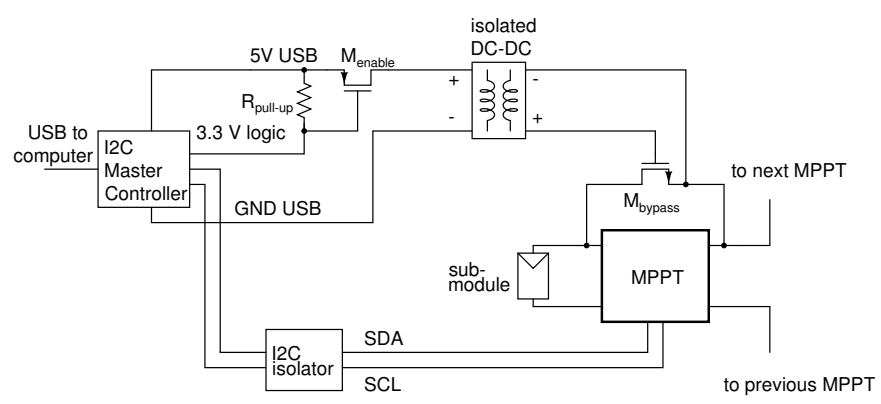

Fig. 7. Schematic drawing of the MPPT bypass circuit which is powered through the USB port, and controlled by a general purpose pin on the I2C host adapter.

electronic load was controlled through the GPIB interface by a small netbook computer that recorded all data.

To effectively characterize the performance benefit of the sub-module distributed power electronics compared to a conventional solar panel, a low on-state resistance bypass MOSFET (PSMN8R5-60YS by NXP) was used, such that the system could be alternated between employing distributed MPPT (MPPTs on, bypass MOSFET off), and conventional operation (MPPTs off, bypass MOSFET on). The bypass MOSFET was connected as shown in Fig. 7, where it can be turned on or off by an isolated dc-dc converter controlled by an enable/disable PMOS driven by a general purpose I/O pin on the USB-connected I2C controller (the Aardvark I2C Host Adapter from Total Phase).

The host adapter provides bidirectional translation of the commands from the USB port to the I2C bus. Custom control software was written in Python to communicate with each MPPT, execute the tracking algorithms, and store data with information about operating voltage, current, and duty cycle of each converter for tracking analysis.

Figure 8 shows an annotated photograph of the circuit board where the bypass circuit, I2C isolation components, and the MPPTs were mounted, together with connectors. Although each circuit board has room for four MPPTs, only three were employed in our experiments, since our solar panel has three bypass diodes.

\section{A. Static Performance Evaluation}

Our first experiment was to evaluate the relative performance improvement offered by the distributed MPPT during a static sub-module mismatch scenario. In this case, we performed measurements with and without distributed MPPT for a panel where a single cell experienced various degrees of shading (as shown in Fig. 9. This scenario is representative of static mismatch caused by for example dirt accumulation, bird droppings, a damaged cell, or a severe local degradation of the panel encapsulant.

Shown in Fig. 10 is a plot of measured panel output power versus load current when a single cell in the panel is shaded by $50 \%$, under constant outdoor insolation (i.e. a short measurement on a cloud-free day). The solid blue line represents the measurement when the panel was connected directly to the electronic load, without distributed MPPTs. In this case, the electronic load was first connected to each individual sub-module, to generate a plot of power versus output current. It can be clearly seen that sub-module 3 has a lower maximum output current (and hence power) due to the single shaded cell.

Furthermore, from the plot showing the full panel power, two maximum power points can be seen. This is due to the bypass diode connected to sub-module 3 conducting when the electronic load is drawing more current than the maximum current available from sub-module 3 . In this case, it can be seen that the global maximum power point is the case where the bypass diode is not conducting, whereas the other point is a local maximum power point. Situations like this present problems for the MPPT algorithms in central and micro-inverters, as they can easily get stuck on the local maximum power point. Table III provides the MPPT tracking parameters used for this and all subsequent MPPT tests. The minimum achievable duty cycle step-size with the hardware we implemented was $0.1 \%$, but the $0.6 \%$ step-size provided a good trade-off between conversion speed and steady-state accuracy.

The green circles in Fig. 10 represent discrete data-points collected with the distributed MPPT converters enabled, for a variety of output currents (stepped by the electronic load). In this measurement, the electronic load stepped the output current to the indicated values with enough time (a few seconds) between steps to ensure that the distributed MPPTs have reached their steady-state points after each step. For this shading scenario (a single cell shaded by $50 \%$ ), a $24 \%$ increase in power output can be observed. The increase in power output is of course dependent on the particular shading pattern (we have measured instances of more than $30 \%$ increase in output power for certain shading patterns). Furthermore, the panel with integrated sub-module MPPTs produces close to its maximum power across a broad range of output currents. In a complete system with a central inverter, this characteristic would enable the inverter to extract maximum power over a wide voltage and current range, rather than the single point associated with a conventional PV system. 


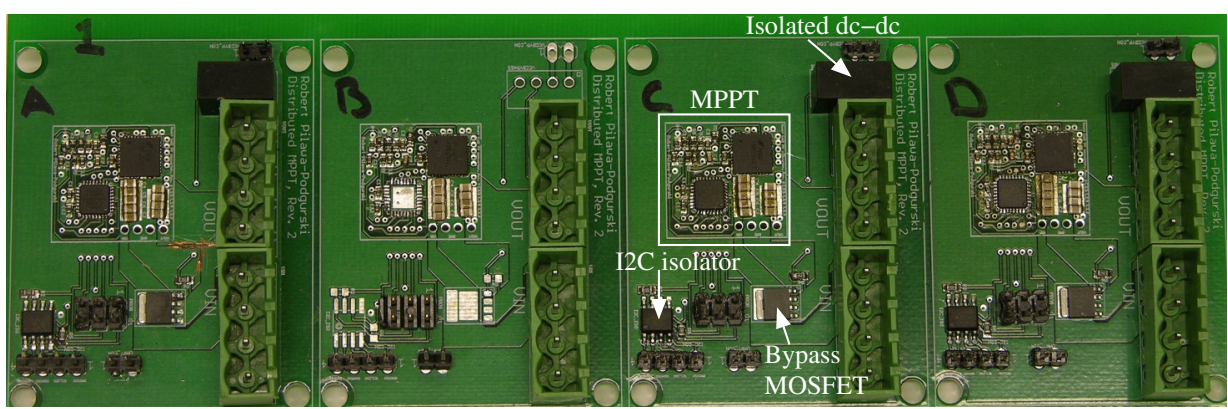

Fig. 8. Annotated photograph of the experimental prototype converters with isolated communication. The bypass MOSFET (powered by the isolated dc-dc converter) enables the MPPT to be bypassed entirely (for evaluation purposes).

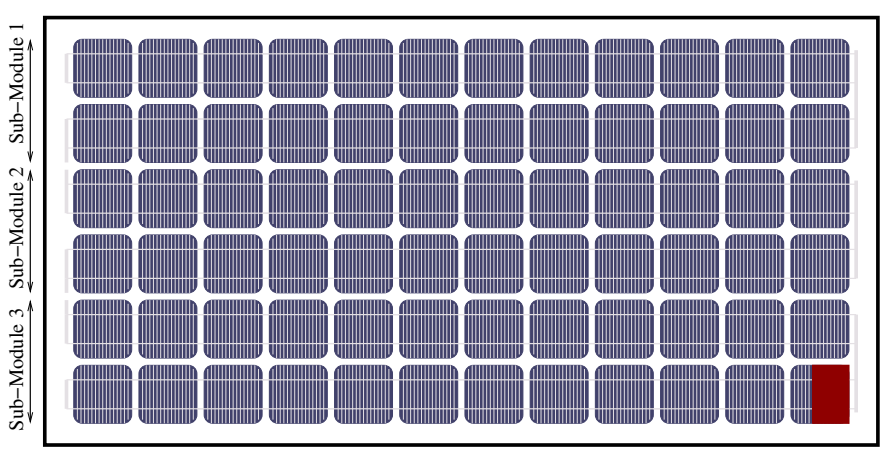

Fig. 9. Drawing of the solar panel illustrating the physical location of the three sub-modules that are accessible through the junction box (corresponding to the electrical wiring schematic shown in Fig. 2). The bottom right cell in Sub-Module 3 is partially shaded in this experiment. The solar panel used in this experiment was the STP175S-24/Ab01 72-cell monocrystalline Si panel from Suntech

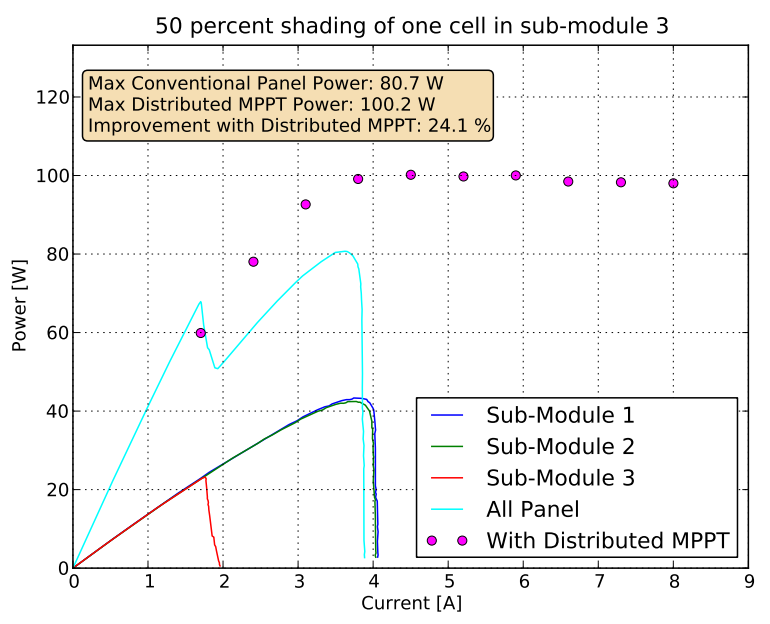

Fig. 10. Plot of power versus current with and without distributed MPPT, for $50 \%$ shading of one cell in sub-module 3 . A power increase of $24 \%$ is observed by the use of the sub-module MPPTs. This data was taken on October 6th, 2011, which was a very sunny day with no cloud coverage.

We have performed a number of measurements on different static scenarios, details of which can be found in [6]. Table IV summarizes these result, where the relative improvement of the distributed MPPT architecture can be clearly seen. For a
TABLE IV

Static Shading Performance

\begin{tabular}{llll}
\hline $\begin{array}{l}\text { Shading of } \\
\text { single cell }\end{array}$ & $\begin{array}{l}\text { Panel power without } \\
\text { sub-module MPPT }\end{array}$ & $\begin{array}{l}\text { Panel power with } \\
\text { sub-module MPPT }\end{array}$ & Change \\
\hline $75 \%$ & $75.2 \mathrm{~W}$ & $83.5 \mathrm{~W}$ & $+11.0 \%$ \\
$50 \%$ & $80.7 \mathrm{~W}$ & $100.2 \mathrm{~W}$ & $+24.1 \%$ \\
$25 \%$ & $103.4 \mathrm{~W}$ & $115.1 \mathrm{~W}$ & $+11.3 \%$ \\
$0 \%$ & $135.5 \mathrm{~W}$ & $132.4 \mathrm{~W}$ & $-2.3 \%$ \\
\hline
\end{tabular}

perfectly matched panel with no shading throughout the day, our proposed system would not be beneficial, as seen from the decrease in output power when employing the distributed MPPT for $0 \%$ shading of a single cell. This should come as no surprise, as any added power electronics incur some loss, and if there is no inherent mismatch in the panel, there is nothing to be gained from employing additional MPPTs. It should be pointed out, however, that it is fairly trivial to implement a bypass-mode in the MPPTs themselves, such that during times of no shading the MPPTs are bypassed altogether, and thus not contributing any loss. This bypass-mode can be implemented in firmware only (turning the top MOSFET on permanently, with some additional conduction loss in the switch and inductor), or with one additional bypass MOSFET with low on-state resistance (this approach will give the lowest loss in no-shading situations).

\section{B. Dynamic Performance Evaluation}

To evaluate the performance of the sub-panel distributed MPPT architecture under dynamic partial shading conditions, we performed the following field experiment under practical real-world conditions:

The panel was placed near a metal chimney, so that only a small number of cells were shaded, as illustrated in Fig. 11. As the sun moves throughout the day, the location of the shadow on the panel moves as well, covering different sections of the panel to varying degrees. This situation is very similar to what would happen in residential installations, where chimneys, power lines, trees, antennas, and other structures block parts of the panel throughout the day.

The system was set up such that approximately every minute it would switch between bypassing the distributed MPPTs, and connecting them to the panel. When the MPPTs are bypassed 


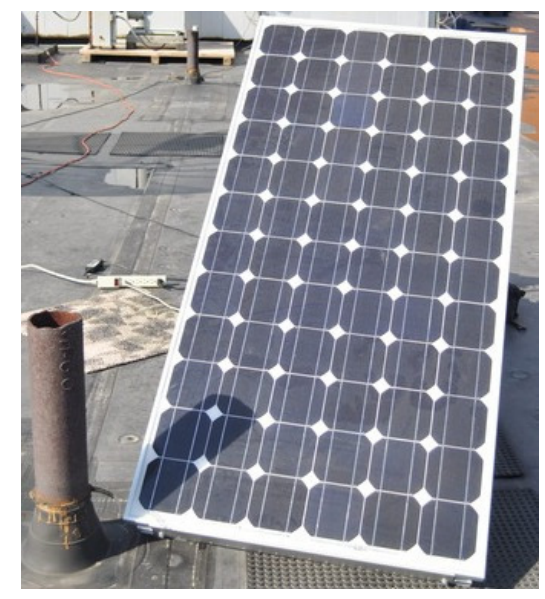

Fig. 11. Photograph illustrating the shading (owing to a protruding pipe) that moves across the panel for the dynamic performance experiment.

(i.e. the panel is configured just like a conventional panel) the electronic load performs a full I-V sweep of the panel, and the highest power is recorded. When the MPPTs are connected, the electronic load starts at a current $(6 \mathrm{~A})$ that is higher than the panel short-circuit current (5.2 A), and waits for the MPPT outputs to reach steady-state (a few seconds). It then decreases the current, at each time waiting for the MPPTs to settle again. It continues to decrease the current until one of the MPPTs (the one connected to the strongest sub-module) reaches its maximum allowed duty cycle (0.99). At this time any further decreases in panel output current will mean that at least one of the MPPTs is not operating at the sub-module MPP, so the sweep is stopped, and the highest output power recorded.

Shown in Fig. 12 is a plot of panel output power versus time, with and without the distributed MPPT electronics, as discussed above. These measurements were taken on a very sunny day $($ Oct 6,2011$)$ at the times indicated in the plot. It can be seen that at all times during the measurement period, the distributed MPPT system generated more power from the panel than what a conventional panel would generate, thanks to the mitigation of sub-module current mismatch owing to partial shading.

Shown in Fig. 13 is the accumulated energy extracted from the panel during the measurement time, and it shows that the distributed MPPT system collects more than $20 \%$ more energy throughout the course of this experiment than what a conventional panel would achieve.

\section{Performance Comparison}

The previous section illustrated the improvements in overall power and energy capture that can be realized with the use of the sub-module MPPT architecture, and the hardware implemented in this work. In solar PV applications, which are very cost sensitive, it is illustrative to perform a cost analysis to quantify the cost-benefit trade-off of this increase in power. A small increase in output power that comes at a large added system cost is clearly not worth it, and in this section we

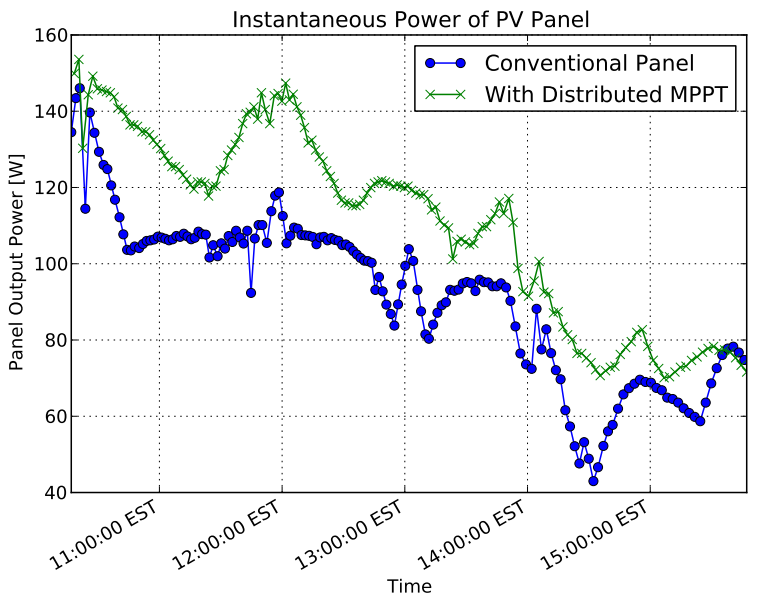

Fig. 12. Instantaneous measured power versus time for a sunny day (October 6, 2011) for a conventional panel, as well as with the distributed MPPT employed. Up to a $30 \%$ increase in captured power is observed.

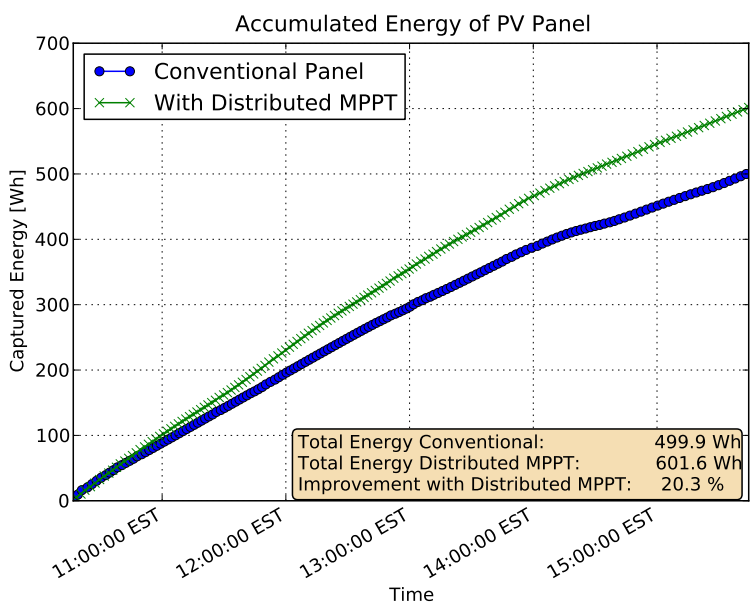

Fig. 13. Accumulated energy versus time for a sunny day (October 6, 2011) for a conventional panel, as well as with the distributed MPPT employed. The distributed MPPT system collects more than $20 \%$ additional energy over a conventional panel.

provide a quantitative analysis of this trade-off, based on the empirical data captured in our experiment.

Shown in Table V is a comparative chart of our work, previous academic work, as well as two selected commercial solutions. The topology, cost, power density, efficiency, and a figure of merit (discussed below) are listed. For the academic work, we have attempted to estimate the complete converter cost from published results ${ }^{3}$ (the commercial prices are estimates from reported retail prices), and adjusted the efficiencies so that they each include all control and gate driver losses for a fair comparison. It should be noted that aside from the work

\footnotetext{
${ }^{3}$ Note that the cost presented in [5] does not include the cost of the microcontroller, gate-driver, auxiliary power supply, and many other components. They are added into the cost used here to provide a fair comparison.
} 
presented here, none of the other solutions provide sub-module tracking, and thus only address mismatch at the panel level. As was shown in the experimental section, sub-module mismatch can contribute to significant energy loss (up to 20\%), which cannot be mitigated by the other solutions. We find that both in terms of efficiency and cost, our solution compares favorably to previously published work in the field, while also offering a significant increase in overall energy capture during partial shading conditions.

\section{Figure OF MERIT}

The merits of distributed MPPT in any solar PV system is entirely dependent of the particular installation. Some installations may benefit greatly from added power electronics, whereas others may see no improvement in overall energy capture (e.g., perfectly matched panels on a completely flat surface with no external objects that can cause shading). Due to the very site-specific circumstances, it is therefore difficult to quantify exactly how much a typical residential installation may benefit from our approach. It is, however, possible to quantify the relative merits of the power electronics itself, compared to other similar solutions. This is done in Table V, where we have introduced a figure of merit that aims to capture some of the cost/benefit trade-off with this approach. It should be pointed out that this figure of merit is a crude estimate of the relative performance between different solutions, and it should not be used as an absolute metric to judge whether distributed MPPT will pay off or not.

The figure of merit attempts to capture the incremental cost for the added average power to the PV system (given as $\$ /$ Watt). It calculates the expected additional average power captured by the system (accounting for the electrical conversion losses of the MPPTs in each case), for a given nominal power increase factor $(\alpha)$. This increase factor represents the fractional increase in average output power that can be expected with the distributed MPPT system, and as such, is highly installation dependent. For our analysis, an $\alpha$ of 0.1 is chosen for per-panel MPPT, and 0.15 for sub-module MPPT (this is a modest 5\% increase for sub-module MPPT compared to per-panel MPPT, keeping in mind that we experimentally measured between a 10\% and 20\% increase in captured energy for the sub-module case versus regular panel-based MPPT in our field experiments). The Figure of Merit is given by:

$$
F O M=\frac{\text { cost }}{\left\langle P_{\text {added }}\right\rangle},
$$

where

$$
\left\langle P_{\text {added }}\right\rangle=\eta_{M P P T} P_{\text {rated }}(1+\alpha)-P_{\text {rated }},
$$

and $\eta_{M P P T}$ is the electrical conversion efficiency of the MPPTs, and $P_{\text {rated }}$ is the rated power of the MPPT. The FOM should be compared to the typical installed cost of solar PV systems, which was estimated to be around $\$ 6 / \mathrm{W}$ in 2010 [11], but is rapidly decreasing. In order for the distributed MPPT system to be cost effective, the FOM must be below the installed cost of the PV system, for a given installation. We see that for our assumptions of a $10 \%$ and $15 \%$ improvement in average power due to module and sub-module tracking, respectively, the cost benefit of many of the solutions of Table V are marginal. As the installed cost of solar PV continues to decrease, even further price pressure on the power electronics is expected. In light of this, our calculated FOM of $0.50 \$ /$ Watt makes our solution cost competitive today, and for some time in the future.

It should be pointed out again that the FOM is highly dependent on the parameter $\alpha$, which attempts to quantify the performance improvements offered by distributed MPPT. It is certainly possible to better quantify this improvement with a more detailed Figure of Merit that models the length of shading (in time), additional panels, and weather data. Our attempt here was merely to elucidate some of the trade-offs in terms of cost and performance, with rough estimates guided from our empirical data.

Finally, we should mention that the FOM described here only captures the monetary value associated with increased energy capture. Distributed power electronics in solar PV installations can provide additional benefits in terms of perpanel (or sub-module) diagnostics and data capture, enabling the user to quickly isolate and replace malfunctioning panels. Distributed power electronics also enables added protection with its ability to completely turn-off the panel output current, something which is not possible with a simple series string architecture. It is expected that these additional services will receive more attention in the future, and may become as important as the increased energy capture.

\section{CONCLUSIONS}

We have presented a sub-module distributed MPPT architecture for solar PV applications, which enables more energy to be extracted from the system. By employing low-voltage synchronous buck converter connected across each sub-module of the panel, a high frequency, very high efficiency power stage can be used. The power electronics can then be miniaturized to the point where they fit into the existing junction box, thereby greatly reducing cost. We have implemented a hardware prototype for use in sub-module tracking of a PV panel, and discuss local and global control techniques to maximize the overall energy capture of the system. We measure up to a $20 \%$ improvement in overall energy capture compared to per-panel MPPT implementation, using field experiments with a partial shading obstacle, and perform static mismatch measurements that further validate the performance of the system. Finally, we compare our implementation to other, state-of-the-art commercial and academic solutions, and find that the proposed solution offers attractive benefits in terms of efficiency and cost, both of which are critical in PV systems.

\section{REFERENCES}

[1] "Trends in photovoltaic applications. survey report of seleted IEA countries between 1992 and 2006.," Tech. Rep. IEA-PVPS T1-16:2007, International Energy Agency Photovoltaic Power Systems, 2007. [Online] www.iea-pvps.org. 
TABLE V

DC-DC OPTIMIZER PERFORMANCE COMPARISON

\begin{tabular}{l|l|l|l|l|l|l}
\hline Work & {$[5]$} & {$[4]$} & {$[12]$} & National & Azuray & This work \\
\hline Type & Academic & Academic & Academic & Commercial & Commercial & Academic \\
\hline Topology & Buck-Boost & Boost & Buck-Boost & Unknown & Unknown & Buck \\
\hline Sub-Module Tracking & No & No & No & No & No & Yes \\
\hline Volume $\left[\mathrm{cm}^{3}\right]$ & $255 \mathrm{~cm}^{3}$ & unknown $($ big) & $850 \mathrm{~cm}^{3}$ & $680 \mathrm{~cm}^{3}$ & $740 \mathrm{~cm}^{3}$ & $12 \mathrm{~cm}^{3}$ \\
\hline Cost & $\$ 27$ & unknown (high) & $\$ 65$ & $\$ 150$ & $\$ 90$ & $\$ 12.80$ \\
\hline Power $[\mathrm{W}]$ & $85 \mathrm{~W}$ & $60 \mathrm{~W}$ & $100 \mathrm{~W}$ & $230 \mathrm{~W}$ & $300 \mathrm{~W}$ & $200 \mathrm{~W}$ \\
\hline Cost/Power $[\$ / \mathrm{W}]$ & $0.32 \$ / \mathrm{W}$ & high & $0.65 \$ / \mathrm{W}$ & $0.65 \$ / \mathrm{W}$ & $0.3 \$ / \mathrm{W}$ & $0.064 \$ / \mathrm{W}$ \\
\hline Efficiency $[\%]$ & $95 \%$ & $93 \%$ & $95 \%$ & $98.5 \%$ & $97.6 \%$ & $98 \%$ \\
\hline FOM $[\$ /($ added W)] & $7.06 \$ / \mathrm{W}$ & & $14.44 \$ / \mathrm{W}$ & $7.81 \$ / \mathrm{W}$ & $4.07 \$ / \mathrm{W}$ & $0.50 \$ / \mathrm{W}$ \\
\hline
\end{tabular}

[2] S. Kjaer, J. Pedersen, and F. Blaabjerg, "A review of single-phase gridconnected inverters for photovoltaic modules," IEEE Transactions on Industry Applications, vol. 41, no. 5, pp. 1292-1306, 2005.

[3] J. Myrzik and M. Calais, "String and module integrated inverters for single-phase grid connected photovoltaic systems - a review," in Proc. IEEE Bologna Power Tech, June 2003.

[4] G. R. Walker and P. C. Sernia, "Cascaded dc-dc converter connection of photovoltaic modules," IEEE Transactions on Power Electronics, vol. 19, no. 4, pp. 1130-1139, 2004.

[5] L. Linares, R. W. Erickson, S. MacAlpine, and M. Brandemuehl, "Improved energy capture in series string photovoltaics via smart distributed power electronics," in Proc. Twenty-Fourth Annual IEEE Applied Power Electronics Conf. and Exposition APEC 2009, pp. 904-910, 2009.

[6] R. C. N. Pilawa-Podgurski, Architectures and Circuits for Low-Voltage Energy Conversion and Applications in Renewable Energy and Power Management. PhD thesis, Department of Electrical Engineering and Computer Science Massachusetts Institute of Technology, 2012.

[7] R. C. N. Pilawa-Podgurski, N. A. Pallo, W. R. Chan, D. J. Perreault, and I. L. Celanovic, "Low-power maximum power point tracker with digital control for thermophotovoltaic generators," in Proc. Twenty-Fifth Annual IEEE Applied Power Electronics Conf. and Exposition (APEC), pp. 961-967, 2010.
[8] D. Perreault and J. Kassakian, "Distributed interleaving of paralleled power converters," IEEE Transactions on Circuits and Systems-Part I: Fundamental Theory and Applications, vol. 44, no. 8, pp. 728-734, 1997.

[9] D. Perreault, J. Selders, R.L., and J. Kassakian, "Frequency-based current-sharing techniques for paralleled power converters," IEEE Transactions on Power Electronics, vol. 13, no. 4, pp. 626-634, 1998.

[10] D. Perreault, K. Sato, J. Selders, R.L., and J. Kassakian, "Switchingripple-based current sharing for paralleled power converters," IEEE Transactions on Circuits and Systems-Part I: Fundamental Theory and Applications, vol. 46, no. 10, pp. 1264-1274, 1999.

[11] G. Barbose, N. Darghouth, R. Wiser, and J. Seel, "Tracking the sun iv - a historical summary of the installed cost of photvoltaics in the united states from 1998 to 2010," tech. rep., Lawrence Berkeley National Laboratory, 2011.

[12] E. Roman, R. Alonso, P. Ibanez, S. Elorduizapatarietxe, and D. Goitia, "Intelligent pv module for grid-connected pv systems," Industrial Electronics, IEEE Transactions on, vol. 53, pp. 1066 -1073, june 2006.

[13] L. Linares, "Design and implementation of module integrated converters for series connected photovoltaic strings," Master's thesis, Unisversity of Colorado, 2009. 